\title{
Role of Polyamines in Ozone Exposed Ischemic-Reperfused Hearts
}

\author{
Rajat Sethi $^{1 \#}$, Sai Raghuveer Chava ${ }^{2}$, Sajid Bashir ${ }^{2,3 \dagger}$, Mauro E. Castro ${ }^{2 \#}$ \\ ${ }^{1}$ Cardiovascular Research and Development Laboratory, Department of Pharmaceutical Sciences, Rangel College of \\ Pharmacy, Texas A \& M Health Science Center, Kingsville, Texas, USA \\ ${ }^{2}$ Department of Chemistry, Texas A \& M University-Kingsville, Kingsville, Texas, USA \\ ${ }^{3}$ Chemical Biology Research Group (CBRG), Kingsville, Texas, USA \\ E-mail: ${ }^{\#}$ rsethi@tamhsc.edu, ${ }^{*} k f m e c 00 @ t a m u k . e d u$
}

Received August 6, 2011; revised September 15, 2011; accepted September 24, 2011

\begin{abstract}
The effect of chronic ozone exposure to ischemia reperfusion $(\mathrm{I} / \mathrm{R})$ injury in isolated perfused rat hearts was previously demonstrated. The present study tested our hypothesis that chronic ozone exposure led to attenuation of polyamines in the heart, which may limit sensitivity to I/R. Sprague Dawley rats were continuously exposed for $8 \mathrm{hrs} /$ day for 28 days to filtered air or $0.8 \mathrm{ppm}$ ozone. Isolated hearts were previously subjected to 0.5 hour of global ischemia followed by 1 hour of reperfusion after which global polyamine content was examined between the two groups. Spermidine production was significantly increased in the experimental group compared to control group (of $\mathrm{I} / \mathrm{R}$ hearts). These results suggest that ozone-induced sensitivity to chronic I/R injury activates myocardial polyamine stress response characterized by increased enzymatic activities and accumulation of spermidine. Collectively, these results suggest that I/R possibly disturbs polyamine metabolism, and increased oxidative stress and concomitant reduced myocardial cell viability.
\end{abstract}

Keywords: Environmental Pollutants, Cardiovascular Disease, Polyamines, Ischemia-Reperfusion (I/R) Injury, Oxidative Stress

\section{Introduction}

Cardiovascular disease is one of the leading causes of mortality in the United States with 81 million reported cases from cardiovascular disease (CVD) in 2006 [1]. Risk factors for CVD are high blood pressure and coronary heart disease (myocardial infarction), which is acute heart attack and angina pectoris (chest pain). Environmental pollutants play an important role in heart disease, as demonstrated by our previous study [2]. Ozone, has been shown to increase heart disease, possibly through oxidation of the plasma membrane resulting in apoptosis of cardiomyocytes [3] or ozone-induced inflammatory response (mediators released into the circulatory system [4]) resulting in organ damage and resultant CVD. Although,

*This work was supported by grants from United States Environmental Protection Agency Grant (USEPA Grant \# IT-83404401-0), Texas A \& M Health Science Research Development Grant (Act\# 134403-35402) and funds from TAMHSC Research Startup (Act\# 13100-35488), Robert A. Welch Foundation (Departmental Grant AC006) and National Science Foundation (NSF) Division of Undergraduate Education (DUE) Program (9987332) for financial support. Dedicated to Karen and Kevin Taylor.

'Dedicated to Karen and Kevin Taylor. the effect of chronic exposure of ozone on CVD was previously investigated and shown to be related to changes in cardiac function after $\mathrm{I} / \mathrm{R}$ injury. This approach measured left ventricular end diastolic pressure (LVEDP) which decreased whereas myocardial tumor necrosis factor-alpha (TNF alpha) and lipid peroxidation levels increased. In addition, superoxide dismutase (SOD) and IL-10 levels decreased in ozone exposed $\mathrm{I} / \mathrm{R}$ hearts compared to $\mathrm{I} / \mathrm{R}$ hearts exposed to filtered air [2]. Collectively, the results suggest that polyamines play a role as possible cardioprotectants to myocardium damage due to chronic ozone exposure [5], which was previously unknown. Polyamines (such as spermidine, cadaverine and putrescine) are linear polycations with one or more amine groups and are involved in cell synthesis of DNA and protein.

These metabolites are catalyzed by catabolic enzymes such as ornithine decarboxylase and spermidine-N1-acetyltransferase which aid synthesis and breakdown respectively. The former also converts ornithine to putrescine and then converts spermidine and spermine in acetylated form, which are converted to putrescine via po- 
lyamine oxidase producing hydrogen peroxide and aminopropionaldehyde in the process [6].

The above metabolite processes could under specific circumstances contribute to cell stress/injury or $I / R$ injury. Furthermore polyamines have been linked to increased susceptibility to hypertrophy; including a positive correlation between intracellular concentration of spermine and calcium attenuation in isolated ventricular myocytes from rats [7]. However, little is known regarding polyamine metabolism and function in myocardial $\mathrm{I} / \mathrm{R}$ injury, particularly during oxidative stress.

Whereas, the oxidant nitric oxide (NO) is known to react with superoxide and generate peroxynitrite anion, also a strong oxidant, the effect of ozone is less known. In addition, it has been documented that TNF alpha induction of NO led to lowering of ODC activity including inhibition of uptake of intracellular polyamines [8]. The degree to which polyamine metabolism is influenced by ozone in $\mathrm{I} / \mathrm{R}$ hearts is unclear. This relationship is the subject of this study with respect to concomitant alterations of cellular polyamines in isolated rat hearts upon $\mathrm{O}_{3}$-induced stress. The contributions of the work remain the same as in the previous study, namely to elucidate the "mechanism of action that results in a decrease in tolerance to myocardial ischemia" [2], however, unlike the previous study focus on utilization of cadaverine as a potential biomarker for oxidative stress. From the proceeding discussion it has been established that the main polyamines involved in cell growth, differentiation and apoptosis are putrescine, spermidine and spermine and that the first rate-limiting enzyme in polyamine biosynthesis is ornithine decarboxylase. It may seem instructive to examine these enzymes and polyamines, however in the current study this was not the case. In our study therefore the focus was measurement of changes in the other polyamines such as cadaverine, putrescine, and spermidine, from which we observed an increase of spermidine in the experimental-to-control ratio; tentatively indicate that a number of triggers are necessary for the appropriate response.

\section{Materials and Methods}

Unless otherwise stated, all chemicals were reagent grade, with ultrapure water. The workflow is summarized in

\section{Figure 1.}

\subsection{Materials and Animals Used}

All chemicals unless otherwise specified where obtained from either Sigma-Aldrich (St. Louis, MO) or VWR International (Chester, PA). The animals Sprague Dawley rats $(50-75 \mathrm{gm})$ were used under conditions previously described [2].

\subsection{Ozone Exposure Conditions}

The procedure used was the same as previously described [2]. Briefly, rats were kept within an environmental chamber supplied with a constant air flow and subjected to ozone as described previously [9].

\subsection{Extraction Procedure}

The procedure used was the same as previously described [10]. Briefly, the frozen heart tissues were weighted and homogenized in isotonic buffer at $\mathrm{pH} 7$ and polyamines extracted as previously described [19].

\subsection{Benzoylation Procedure}

The polyamines were isolated from the homogenized heart tissue through centrifugation and were derivatized as previously described [11]. Benzoylated polyamines were stored at $-20^{\circ} \mathrm{C}$ in all these previous methods.

\subsection{HPLC Method}

The method used was the same as previously described [11]. Here, the solvent system was methanol (solvent A) and water (solvent $\mathrm{B}$ ) with a flow rate $1.0 \mathrm{ml} / \mathrm{min}$ (method I) was throughout the isocratic method and the chromatograph for the experimental group is shown in Figure 2.

The benzoylated amines were extracted with diethyl ether, which were eluted at room temperature through a $4.6 \times 250 \mathrm{~mm}, 5 \mu \mathrm{m}$ particle size reverse-phase $\mathrm{C}_{18}$ column which is detected at $254 \mathrm{~nm}$. The time required for completing one single run was 20 minutes and is shown in Figure 3.

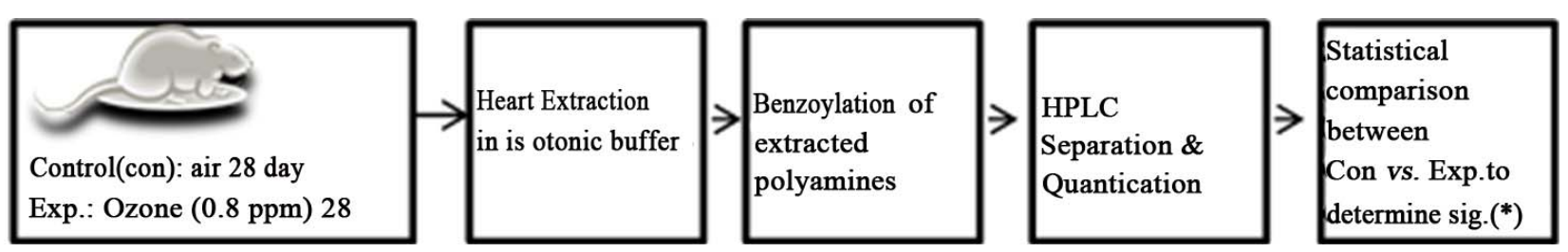

Figure 1. Workflow used in the experimental phase. 


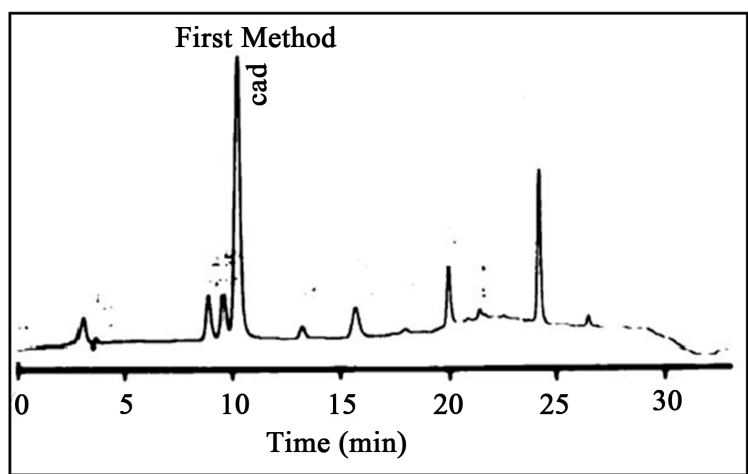

Figure 2. HPLC Chromatogram for procedure I for separation of polyamines (40 minutes run).

An alternative method described by Schotten-Bauma$\mathrm{nn}$ benzoylation procedure (procedure III, cited in [11]) was also used as a comparison to our chromatographic procedure (Figure 4).

\subsection{Statistical Analysis}

All the data are presented as mean \pm standard error (S.E.). The biosynthesis and degradation processes of the different polyamines are connected, the relationship with intracellular levels of these polyamines could be assessed using either one-way or two-way ANOVA was used to compare differences among groups where appropriate. A two-way ANOVA could give us this information. To differentiate between ozone-exposure hearts compared with the control hearts Student's t-test were performed. Statistical comparison was performed by paired or unpaired Students t-test. Significance level was setup at $P<$ 0.05 . The linear regression analysis was used to determine the correlation between different variables. In some cases, ad hoc test was used.

\section{Results and Discussion}

Figures 2-3 show chromatograms for the standard polyamines extracted from the control group. All chromatograms (Figures 2-7) demonstrate baseline separation and quantification of each polyamine cross-referenced to authentic standards.

The chromatographs from experimental group were compared with standards to identify specific polyamines (Figures 5-6 of chromatograms corresponding to experimental group) which were identified and quantified.

The three polyamines were normalized for per gram of fresh-weight heart tissue and compared and contrasted with the control group. In addition, a statistical test was undertaken to determine whether these differences were statistically significant.

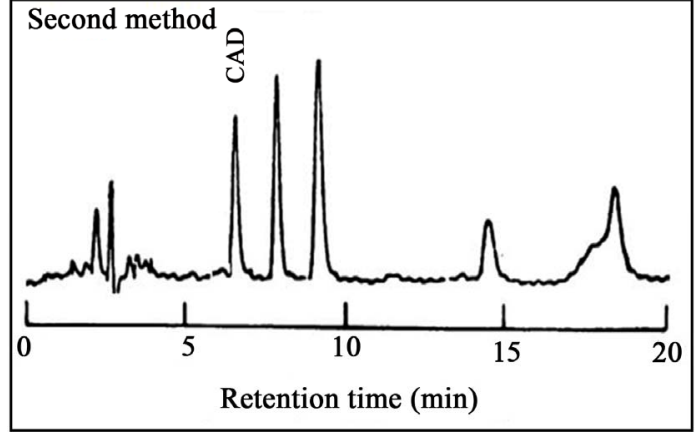

Figure 3. HPLC Chromatogram for procedure II (same as I except chloroform was used in the extraction step instead of diethyl ether) for separation of polyamines (20 minutes run).

The comparison is shown in Figure 7 and for cadaverine and spermidine the differences between the experimental and control group were significant at the $95 \%$ level $(P<0.05)$ except for putrescine, due to biological variation. To assess whether this variation was higher than expected, a literature survey of standard error means for other similar systems was examined and summarized in Table 1.

Table 1 summarizes SE (Standard Error) values from literature (research) in comparison to values from present research. The significance of the results is discussed below. The putrescine samples were diluted to allow accurate measurements of cadaverine and spermidine.

From the analysis it appears that the biological variation in our study is comparable to other studies and not an experimental artifact.

The results in Table 1 indicate the biological fluctuations in polyamine content are within the general error envelope indicated in other studies. The results also indicate that during oxidative damage/stress, for example ischemia, activities of the metabolite enzymes presumably ornithine decarboxylase spermidine/spermine N1-acetyltransferase, polyamine oxidase are altered due to

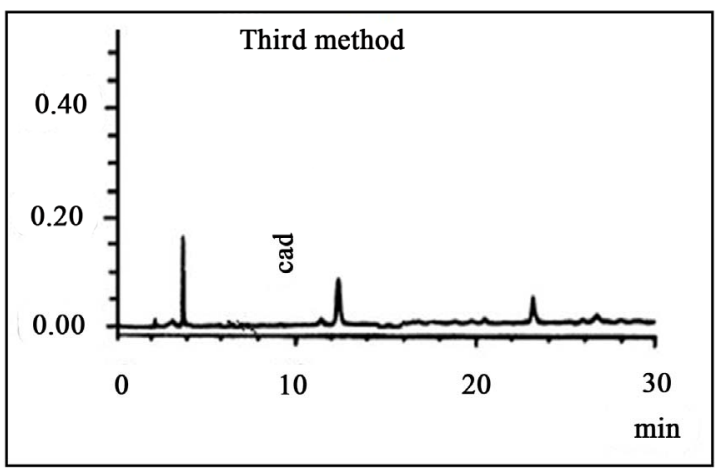

Figure 4. HPLC Chromatogram for procedure III for separation of polyamines 20 minutes run (same was I except with heated column). 


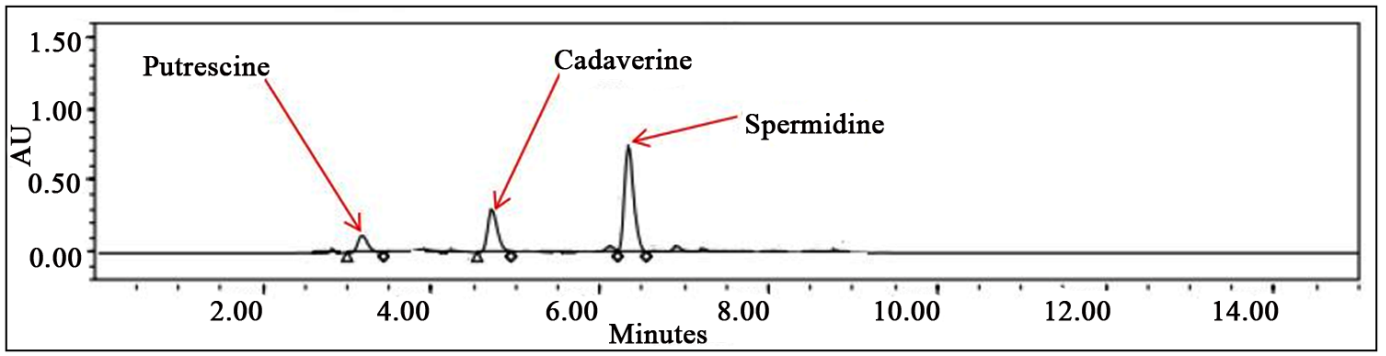

Figure 5. HPLC Chromatogram of experimental group with polyamines extracted and diluted with buffer at 1:8 ratio of polyamine:buffer (with putrescine dilution, procedure I).

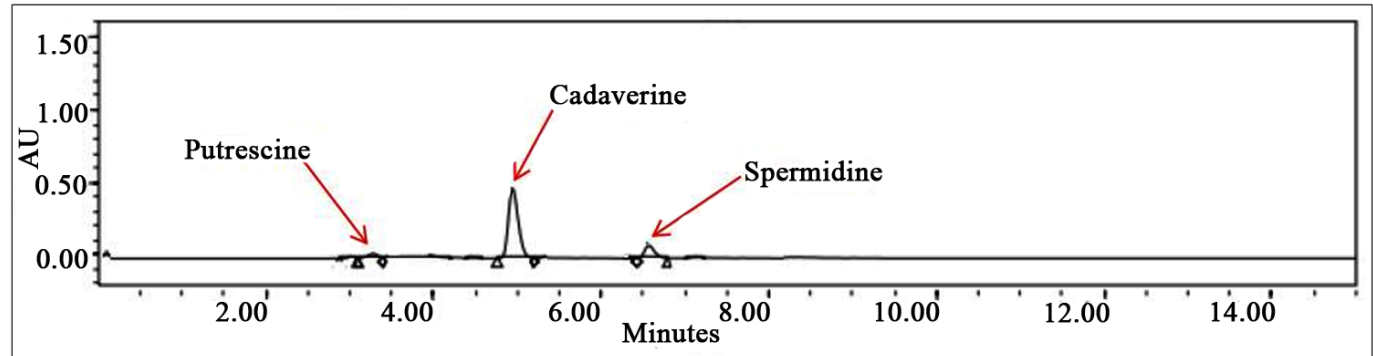

Figure 6. HPLC Chromatogram control group extracted and diluted with buffer at 1:8 ratio of polyamine:buffer (with putrescine dilution, procedure I).

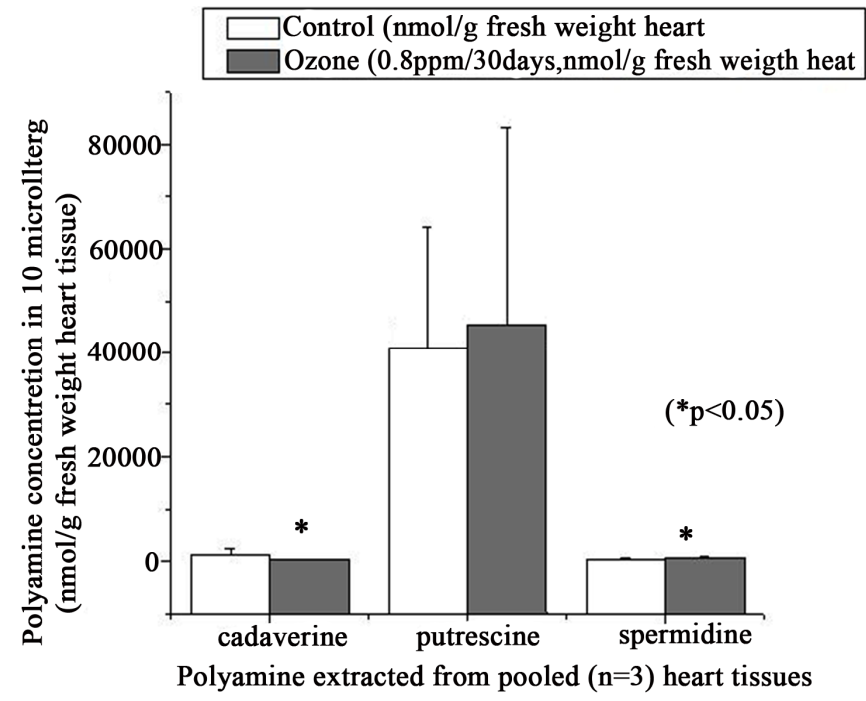

Figure 7. Plot of polyamine pool isolated from rat heart tissue corresponding to control group (white) and experimental group (grey) with ANOVA statistical analysis at $95 \%$ significance $(*)$ indicating that alterations of indigenous pool was statistically significant for cadaverine and spermidine, of which the latter may confer some protection against I/R injury.

Table 1. Percentage of SE in polyamine values from previous researches and present research values.

\begin{tabular}{ccccccccc}
\hline \multicolumn{2}{c}{$\%$ of SE in Cadaverine } & \multicolumn{2}{c}{$\%$ of SE in Putrescine } & \multicolumn{2}{c}{$\%$ of SE in Spermidine } & \multicolumn{2}{c}{ Others Works } & Reference \\
\hline Control & Test & Control & Test & Control & Test & Control & Test & \\
28.12 & 23.43 & 18.56 & 27.84 & 12.51 & 21.92 & ND & ND & Our Results \\
ND & ND & $31.20^{1}$ & $32.52^{1}$ & $18.37^{1}$ & $15.28^{2}$ & $11.99^{1}$ & $22.30^{3}$ & 19 \\
ND & ND & 35.89 & 65.18 & 17.77 & 17.83 & $34.94^{3}$ & $24.68^{3}$ & 20 \\
ND & ND & 14.28 & 30.4 & 26.09 & 36.07 & $20^{3}$ & $25^{3}$ & 21 \\
ND & ND & 14.62 & 22.63 & 14.12 & 17.83 & $12.26^{3}$ & $19.48^{3}$ & 22 \\
ND & ND & 28 & 59.25 & 8.06 & 44.77 & $15.81^{3}$ & $38.22^{3}$ & 23 \\
\hline
\end{tabular}


induction triggered by ozone $\left(\mathrm{O}_{3}\right)$ induced toxicity as reflected in the measured concentrations. Putrescine levels were increased, but were not statistically significant; however, statistically significant were cadaverine levels which decreased and spermidine levels which increased. It is known the polyamine homoeostasis is dependent upon ornithine decarboxylase, spermidine/spermine N1 -acetyltransferase and to a lesser degree lysine decarboxylase, depending upon the up-regulation of ornithine decarboxylase, spermidine/spermine N1-acetyl-transferase, whereby changes in enzyme activity would in turn affect putrescine content. We speculate putrescine levels to decrease because if spermine N1-acetyltransferase were down-regulated instead, then the expected increase in putrescine would be marginal as observed in our study, in other words any increase in the synthesis of cadaverine by ornithine decarboxylase is offset by conversion to spermidine by spermidine synthase and polyamine oxidase, leading to an expected increase of spermidine which was also observed. The magnitude of the polyamine would depend on the duration and strength of the external stimuli [12]. The polyamine metabolism changes as observed in the heart in the present study might be similar yet distinct to what has been observed in polyamine alterations in cerebral ischemia of polyamine stress response in brain [13]. These biological alterations may be implicated in ischemia-related cell injury in heart tissue. Free radical oxidant production and calcium ion loading are two mechanisms implicated in the development of myocardial $\mathrm{I} / \mathrm{R}$ injury. As a result of $\mathrm{O}_{3}$ induced injury spermidine levels increased to a greater exten than putrescine levels which either increased slightly or remained essentially constant indicating that polyamine synthesis pathway was down-regulated and polyamine degradation pathway was up-regulated. Ornithine decarboxylase is a rate-limited enzyme in the synthesis of putrescine from ornithine and is susceptible to direct oxidation, [14] leading to inactivation.

Similarly, spermidine/spermine N1-acetyltransferase catalyzes the acetylation of putrescine to spermidine, which upon induction by various NO or toxins or stress, in conjugation with polyamine oxidase could lead to a decrease of spermine and production of hydrogen peroxide and aldehyde by-product.

Up-regulation may decrease spermine mitigating oxidative stress response. However, a decrease in spermine levels may not correspond to a similar decrease in spermidine levels, since the latter can also be synthesized by spermidine synthase affecting other biological systems regulated by polyamines. Systems regulated by polyamines include free radical scavenging and reduction in lipid peroxidation [15].

Although speculative, up-regulation of spermidine/ spermine N1-acetyltransferase could lead to the generation of hydrogen peroxide and aldehydes in ischemic hearts and alterations to spermine and possible spermidine $(\uparrow)$ and calcium ions $(\uparrow)$, ameliorating any positive changes in protection, [16] through DNA base-pair stabilization from oxidative stress, or protection from endonucleases.

This is because there is a body of literature which connects I/R injury to alterations in spermine [17] and stress in a number of animal/plant models including use of difluoromethylornithine (DFMO) as a potent inhibitor of polyamine biosynthesis, Ornithine decarboxylase (ODC) [18], therefore I/R injury and alterations in ROS and/or NO levels and quantification of apoptosis in the different groups has been established as a leading hypothesis in the mechanism of injury via spermine/ODC alterations.

Once the mechanism is trigged an increases in spermidine levels can provid a cardioprotective effect. This protection from oxidative damage from $\mathrm{O}_{3}$-induced stress may be through cell membrane stabilization and increased scavenging of oxygen radical species in addition to preventing calcium ion loading [18].

Consistent with earlier observations from our previous study that both malondialdehyde and TNF alpha levels increased with decreases in interlukin-10 (IL-10) levels is indicative of increased myocardial oxidative stress levels [2].

$\mathrm{O}_{3}$-mediated lipid peroxidation is counted by radical scavengers, such as SOD or spermidine through binding to membrane phospholipids or formation of a polycationcomplex through modification of the auto oxidation of $\mathrm{Fe}^{2+}$ [19] leading to inhibition of lipoxygenase, there- by minimizing peroxidative damage. Since SOD is a key enzyme for detoxification of superoxide anions, generated by oxidants such as $\mathrm{O}_{3}$, lowering of SOD/glutathione reductase and glutathione S-transferase glutathione activity (free glutathione) may be countered by polyamines such as spermidine/spermine, which has been demonstrated in plants [20] including O3-induced leaf necrosis in tobacco [21]. Our model consistent with our previous findings and current study indicates that upon generation ozone TNF alpha is induced leading to generation of reactive oxygen species (ROS) induced apoptosis through activation of JNK [22]. In response to this stress, the cell utilizes anti-oxidants such as glutathione, or antioxidant enzymes such as SOD. Concurrent to SOD expression, polyamines, particularly spermidine/spermine reduce TNF alpha-induced apoptosis. It has also been shown that JNK and $\mathrm{p} 38$ proteins (proapoptotic mediators from the MAPK family of proteins) are activated during UV/oxidative stress [23]. From the above outline, it can be summarized that JNK activation, either directly 
or indirectly via proapoptotic mediator by TNF alpha would lead to apoptosis. Inhibition I/R-induced oxidative stress of TNA alpha by IL-10 as a first response (early phase), plus effect of glutathione, SOD (early/intermediate phase) and alterations in polyamine content (e.g. depletion of cadaverine, increase in intermediate/late phase) confer a limited degree of protection. The effect of polyamines on JNK inactivation through increased ERK (antiapoptotic mediators from the MAPK family of proteins) through dephosphorylation. The protective effects of ERK in cardiomyocytes have already been documented [24] through the Bcl-2 family of proteins [25] with release of cytochrome c, although in our study this was not investigated.

\section{Conclusions}

In summary, prolonged exposure to ozone gave rise to increased putrescine (approximately 1.06-fold relative to control) and spermidine (1.57-fold) concentration, and decreased cadaverine (4.54-fold) concentration. From the above results spermidine levels may be compensating the changes in putrescine and cadaverine, but only up to certain levels. The results were pair-wise compared and were found to be significant for cadaverine and spermidine with biological variation within other published literature values.

These results indicate that $\mathrm{O}_{3}$ can activate myocardial polyamine stress response pathway(s) resulting in enhanced I/R injury. This injury can be attributed to alterations in cadaverine and putrescine concentrations in the myocardium. We believe that the increase in spermidine levels seen in our study may be involving a compensatory response to $\mathrm{O}_{3}$ induced ischemic injury. The mechanism of depletion may activate certain pathways, such as inhibition of TNF alpha, which in turn prevents JNK activation and promotes apoptosis via MAPK family of proteins. These proteins regulate apoptotic signaling via ERK activation, which has been observed in other studies.

Lastly, we believe that indigenous spermidine stabilizes the intracellular polyamine pool which in turn provides limited protection against I/R injury.

\section{Acknowledgements}

We are grateful to Mohammad T. Nutan (TAMHSC) for giving permission to use the HPLC and Mr. Don Marek from the Department of Environmental Engineering (TAMUK) for assistance with the glove box.

\section{References}

[1] T. Thom, N. Haase, W. Rosamond, V. J. Howard, J. Rumsfeld, T. Manolio, Z. J. Zheng, K. Flegal, C. O’Donnell,
S. Kittner, D. Lloyd-Jones, D. C. Goff, Y. Hong, R. Adams, G. Friday, K. Furie, P. Gorelick, B. Kissela, J. Marler, J. Meigs, V. Roger, S. Sidney, P. Sorlie, J. Steinberger, S. Wasserthiel-Smoller, M. Wilson and P. Wolf, "Heart Disease and Stroke Statistics-2006 Update: A Report from the American Heart Association Statistics Committee and Stroke Statistics Subcommittee," Circulation, Vol. 113, 2006, pp. E85-E151. doi:10.1161/CIRCULATIONAHA.105.171600

[2] R. S. P. Perepu, C. Garcia, D. Dostal and R. Sethi, "Enhanced Death Signaling in Ozone Exposed IschemicReperfused Hearts," Molecular and Cellular Biochemistry, Vol. 336, No. 1-2, 2010, pp. 55-64. doi:10.1007/s11010-009-0265-4

[3] K. K. Sathish, M. Haque, T. E. Perumal, J. Francis and R. M. Uppu, "A Major Ozonation Product of Cholesterol, 3Beta-Hydroxy-5-Oxo-5, 6-Seco-Cholestan-6-al, Induces Apoptosis in H9c2 Cardiomyoblasts," FEBS Letters, Vol. 579 , No. 28, 2005, pp. 6444-6450. doi:10.1016/j.febslet.2005.10.044

[4] R. D. Brooke, "Cardiovascular Effects of Air Pollution," Clinical Science, Vol. 115, 2008, pp. 175-187. doi:10.1042/CS20070444

[5] J. B. Ruidavets, M. Cournot, S. Cassadou, M. Giroux, M. Meybeck and J. Ferrieres, "Ozone Air Pollution Is Associated with Acute Myocardial Infarction," Circulation, Vol. 111, 2005, pp. 563-569. doi:10.1161/01.CIR.0000154546.32135.6E

[6] K. Niiranen, M. Pietilä, T. J. Pirttilä, A. Järvinen, M. Halmekytö, V. P. Korhonen, T. A. Keinänen, L. Alhonen and J. Jänne, "Targeted Disruption of Spermidine/Spermine N1-Acetyltransferase Gene in Mouse Embryonic Stem Cells. Effects on Polyamine Homeostasis and Sensitivity to Polyamine Analogues," Journal of Biological Chemistry, Vol. 277, 2002, pp. 25323-25328. doi:10.1074/jbc.M203599200

[7] R. Wang, C. Q. Xu, W. M. Zhao, J. Zhang, K. Cao, B. F. Yang and L. F. Wu, "Calcium and PolyaminE Regulated Calcium-Sensing Receptors in Cardiac Tissues," European Journal of Biochemistry, Vol. 270, No. 12, 2003, pp. 2680-2688. doi:10.1046/j.1432-1033.2003.03645.x

[8] J. Satriano, S. Ishizuka, D. C. Archer, R. C. Blantz and C. J. Kelly, "Regulation of Intracellular Polyamine Biosynthesis and Transport by NO and Cytokines TNF-Alpha and IFN-Gamma," American Journal of Physiology, Vol. 276 (4 Pt 1), 1999, pp. C892-C899.

[9] M. J. Wiester, J. S. Tepper, M. E. King, M. G. Menache and D. L. Costa, "Comparative Study of Ozone $\left(\mathrm{O}_{3}\right)$ Uptake in Three Strains of Rats and in the Guinea Pig," Toxicology and Applied Pharmacology, Vol. 96, No. 1, 1998, pp. 140-146. doi:10.1016/0041-008X(88)90256-6

[10] R. Sethi, N. S. Dhalla, "Inotropic Responses to Isoproterenol in Congestive Heart Failure Subsequent to Myocardial Infarction in Rats," Journal of Cardiac Failure, Vol. 1, No. 5, 1995, pp. 391-399. doi:10.1016/S1071-9164(05)80008-9 
[11] R. Sethi, S. R. Chava, S. Bashir and M. E. Castro, "An Improved High Performance Liquid Chromatographic Method for Identification and Quantization of Polyamines as Benzoylated Derivatives," American Journal of Analytical Chemistry, Vol. 2, 2011, pp. 456-469. doi:10.4236/ajac.2011.24055

[12] Y. Hayashi, J. Tanaka, Y. Morizumi, Y. Kitamura and Y. Hattori, "Polyamine Levels in Brain and Plasma after Acute Restraint or Water-Immersion Restraint Stress in Mice," Neuroscience Letters, Vol. 355, No. 1-2, 2004, pp. 57-60. doi:10.1016/j.neulet.2003.10.027

[13] B. A. Coert, R. E. Andereson and F. B. Meyer, "Exogenous Spermine Reducesischemic Damage in a Model of Focal Cerebral Ischemia in the Rat," Neuroscience Letters, Vol. 282, No. 1-2, 2000, pp. 5-8. doi:10.1016/S0304-3940(00)00856-9

[14] J. M. Dypbukt, M. Ankarcrona, M. Burkitt, A. Sjoholm, K. Strom, S. Orrenius and P. Nicotera, "Different Prooxidant Levels Stimulate Growth, Trigger Apoptosis, or Produce Necrosis of Insulin-Secreting RINm5F Cells," Journal of Biological Chemistry, Vol. 269, 1994, pp. 30553-50560.

[15] K. C. Das and H. P. Misra, "Hydroxyl Radical Scavenging and Singlet Oxygen Quenching Properties of Polyamines," Molecular and Cellular Biochemistry, Vol. 262, No. 1-2, 2004, pp. 127-133. doi:10.1023/B:MCBI.0000038227.91813.79

[16] J. M. Ribeiro and D. A. Carson, " $\mathrm{Ca}^{2+} / \mathrm{Mg}^{2+}$-Dependent Endonuclease from Human Spleen: Purification, Properties and Role in Apoptosis," Biochemistry, Vol. 32, No. 35, 1993, pp. 9129-9136. doi:10.1021/bi00086a018

[17] G. Drolet, E. B. Dumbroff, R. L. Legge and J. E. Thompson, "Radical Scavenging Properties of Polyamines," Phytochemistry, Vol. 25, No. 2, 1986, pp. 367-371. doi:10.1016/S0031-9422(00)85482-5

[18] R Alcázar, T. Altabella, F. Marco, C. Bortolotti, M. Reymond, C. Koncz, P. Carrasco and A. F. Tiburcio, "Polyamines: Molecules with Regulatory Functions in Plant Abiotic Stress Tolerance," Planta, Vol. 231, 2008, pp. 1237-1249.
[19] G. Noctor, A. M. Arisi, L. Jouanin, K. J. Kunert, H. Rennenberg and C. Foyer, "Glutathione: Biosynthesis, Metabolism and Relationship to Stress Tolerance Explored in Transformed Plants," Journal of Experimental Botany, Vol. 49, 1988, pp. 623-647. doi: $10.1093 /$ jexbot $/ 49.321 .623$

[20] C. Langebartels, K. Kerner, S. Leonardi, M. Schraudner, M. Trost, W. Heller and H. Sandermann, "Jr. Biochemical Plant Responses to Ozone. Differential Induction of Polyamine and Ethylene Biosynthesis in Tobacco," Plant Physiology, Vol. 95, 1991, pp. 882-889. doi:10.1104/pp.95.3.882

[21] Z. G. Liu, H. Hsu, D. V. Goeddel and M. Karin, "Dissection of TNF Receptor 1 Effector Functions: JNK Activation Is Not Linked to Apoptosis While NF-kB Activation Prevents Cell Death," Cell, Vol. 87, No. 3, 1996, pp. 565576. doi:10.1016/S0092-8674(00)81375-6

[22] J. M. Kyriakis and J. Avruch, "Protein Kinase Cascades Activated by Stress and Inflammatory Cytokines," Bioessays, Vol. 18, No. 7, 1996, pp. 567-577. doi:10.1002/bies.950180708

[23] R. Aikawa, I. Komuro, T. Yamazaki, Y. Zou, S. Kudoh, M. Tanaka, I. Shiojima, Y. Hiroi and Y. Yazaki, "Oxidative Stress Activates Extracellular Signal Related Kinases through Src and Ras in Cultured Cardiac Myocytes of Neonatal Rats," Journal of Clinical Investigation, Vol. 100, No. 7, 1997, pp. 1813-1821. doi:10.1172/JCI119709

[24] M. Mori, M. Uchida, T. Watanabe, K. Kirito, K. Hatake, K. Ozawa and N. Komatsu, "Activation of Extracellular Signal Related Kinases ERK1 and ERK2 Induces Bcl-xL up-Regulation via Inhibition of Caspase Activities in Erythropoietin Signaling," Journal of Cell Physiology, Vol 195, No. 2, 2003, pp. 290-297. doi:10.1002/jcp.10245

[25] T. E. Soderstrom, M. Poukkula, T. H. Holmstrom, K. M. Heiskanen and J. E. Eriksson, "Mitogen-Activated Protein kinase/Extracellular Signal-Related Kinase Signaling in Activated T Cells Abrogates TRAIL-Induced Apoptosis Upstream of the Mitochondrial Amplification Loop and Caspase-8," Journal of Immunology, Vol. 169, 2002, pp. 2851-2860. 


\section{Author Contribution}

S. R. Chava undertook all of the experimental work (extraction, triplicate analysis) related to the chromatography. S. Bashir assisted in the extraction and the first set of analyses. He wrote the first and co-wrote the second and submission draft of the manuscript. M. Castro and R. Sethi co-supervised the student towards his Master of Science thesis. R. Sethi undertook the entire animal component and wrote the first paper (Ref [2]) and conferred on the current submitted draft. Finally, Dr. Castro assisted with the publication costs.

\section{Abbreviations}

$\begin{array}{ll}\text { ANOVA } & \text { Analysis Of Variance } \\ \text { Bcl-2 } & \text { B cell leukaemia-2 } \\ \text { CD20 } & \text { Cluster of differentiation twenty } \\ \text { Cad } & \text { Cadaverine } \\ \text { DFMO } & \text { Difluoromethylornithin } \\ \text { CVD } & \text { Cardiovascular disease } \\ \text { DNA } & \text { Deoxyribonucleic acid }\end{array}$

ERK

$\mathrm{Fe}^{2+}$

HPLC

$\mathrm{I} / \mathrm{R}$

IL-10

JNK

LVEDP

MALT

MAPK

ND

$\mathrm{NO}$

$\mathrm{O}_{3}$

ODC

P-value

Put

ROS

SOD

Student t-test Testing the difference between means

TNF alpha Tumor necrosis factor-alpha

UV

Extracellular signal-regulated kinase pathway protein Iron (II) cation High performance liquid chromatography Ischemia reperfusion Interleukin-10 c-Jun NH2-terminal protein kinases Left ventricular end diastolic pressure Mucosa associated lymphoid tissue Mitogen activated protein kinase Not determined Nitric oxide

\section{Ozone}

Ornithine decarboxylase

Probability value (estimation of Randomness)

\section{Putrescine}

Reactive oxygen species Superoxide dismutase

Ultraviolet 